\title{
High dose steroids in acute relapses of multiple sclerosis: MRI evidence for a possible mechanism of therapeutic effect
}

\author{
D H Miller, A J Thompson, S P Morrissey, D G MacManus, S G Moore, B E Kendall, \\ I F Moseley, W I McDonald
}

\begin{abstract}
The integrity of the blood-brain barrier (BBB) in multiple sclerosis (MS) was monitored by serial gadolinium-(Gd)DTPA enhanced MRI during and after the treatment of acute relapses with a three day course of high dose intravenous methylprednisolone (IVMP). During treatment there was a rapid reduction of BBB abnormalities in $96 \%$ of enhancing lesions. In spite of sustained clinical improvement, many lesions re-enhanced within a few days of stopping IVMP, and new lesions frequently appeared within one month. It is possible that the rapid, albeit transient, reversal of BBB abnormalities contributes to the accelerated recovery from relapses associated with IVMP treatment.
\end{abstract}

A short course of high dose intravenous methylprednisolone (IVMP) is widely used to treat clinical relapses in multiple sclerosis (MS). There is evidence to suggest that IVMP, like ACTH, accelerates recovery from relapses. ${ }^{1-6}$

The mechanism of action of IVMP, as of other forms of steroids, is uncertain. Ultimately, it must improve compromised nerve conduction through the lesion. Suggestions as to how this may be achieved have included the reduction of oedema ${ }^{7}$ immunosuppression, $^{8}$ direct inhibition of demyelination, ${ }^{9}$ a direct effect on axonal conduction, and reversal of blood-brain barrier (BBB) abnormalities. A breakdown of the $\mathrm{BBB}$ in association with inflammation is the earliest detectable event in the development of the new lesion in MS. ${ }^{10}$ Serial iodine enhanced CT scanning has shown that IVMP may rapidly reverse BBB abnormalities in MS lesions. ${ }^{11}$ However, the consistency and duration of this effect can not be defined on CT because of the unacceptability of frequent scanning, and the low sensitivity of this modality to MS lesions.

BBB impairment in MS lesions can now be readily detected and safely monitored by gadolinium (Gd)-DTPA enhanced MRI. ${ }^{12}{ }^{13}$ Using this technique we have studied the effect of IVMP on BBB abnormalities in MS lesions to assess its relevance as a therapeutic mechanism.

\section{Methods}

Ten patients were recruited, nine with clinically definite $\mathrm{MS},{ }^{14}$ and one with an isolated acute demyelinating spinal cord syndrome (the latter patient had multiple white matter lesions on brain MRI and IgG oligoclonal bands in the CSF). There were nine females and one male, aged between 24-42 years (mean age 30.6 years). Disease duration (in the nine with clinically definite MS) ranged from five months to 10 years (mean 5.8 years). All were being treated with IVMP because of a clinical relapse of between one to six weeks duration; the relapse involved the spinal cord in nine patients and the brainstem in one.

The patients received IVMP $1 \mathrm{~g}$ a day for three days. Each had a total of five MRI brain scans at the following times: 1) Less than 24 hours before the first dose of IVMP; 2) After the first dose of IVMP; 3) At the end of the three day course of treatment; 4) One week after the start of treatment; 5) Approximately one month after the start of treatment (mean 4.8 weeks, range 4-7 weeks).

Brain MRI was performed on a Picker 0.5T superconducting scanner in the following manner: 1) Pre-contrast: a $T_{2}$-weighted sequence $\left(\mathrm{SE}_{2000 / 60}\right)$ was obtained with 24 contiguous, 5 mm thick, axial slices; 2) Post-contrast: imaging was begun 10 minutes after intravenous injection of Gd-DTPA $0 \cdot 1 \mathrm{mmol} / \mathrm{kg}$. $T_{1}-$ weighted images were obtained $\left(\mathrm{SE}_{500 / 20}\right)$; the whole brain was surveyed in three sets each consisting of eight contiguous, $5 \mathrm{~mm}$ thick axial slices, which were obtained in the same order at each follow up.

In three of the patients who had had a recent spinal cord relapse, pre-contrast $T_{2}$-weighted $\left(\mathrm{SE}_{1500 / 80}\right)$ and post contrast $\mathrm{T}_{1}$-weighted $\left(\mathrm{SE}_{500 / 20}\right)$ scans were obtained through the symptomatic lesion with contiguous, $5 \mathrm{~mm}$ thick, axial and sagittal slices, using a surface receiver coil. Because of time constraints, the spinal cord was not examined in the six other patients with a recent spinal cord relapse.

MRIs were reported by two neuroradiologists (BK, IM). They first identified all enhancing lesions which were visible on the pretreatment scan. These lesions were then followed through subsequent scans, and on each occasion the intensity and extent of persisting enhancement was compared with that seen in the pre-treatment lesion. At follow up, there were four possible outcomes for both intensity and extent of enhancement in every lesion, that is, it either no longer enhanced, or the enhancement was decreased, unchanged or increased in intensity or extent compared with the pretreatment scan (table 1).

Follow up $T_{1}$ - and $T_{2}$-weighted scans were analysed for the presence of new disease 

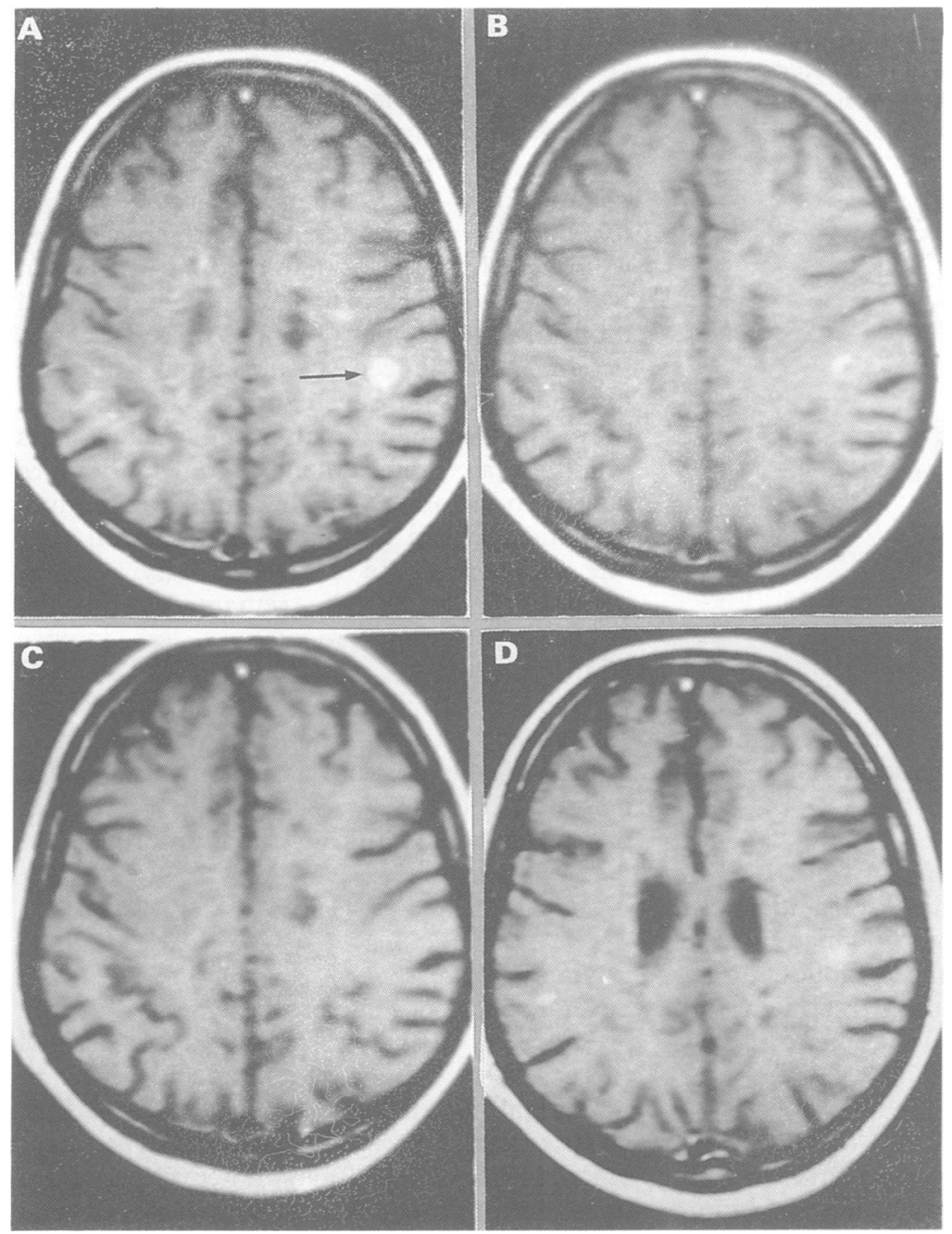

Figure 1 Serial Gd-DTPA enhanced $T$,-weighted MRI (SE $\left.E_{\text {soizo }}\right)$. Before treatment with IVMP (a), there is a prominent, round, enhancing lesion in the right parietal white matter (arrow). The lesion shows much reduced enhancement after one day of IVMP (b), and no enhancement after three days (c). After one week (d), the lesion re-enhances, although to a lesser degree than was the case prior to treatment. Although image (d) is at a slightly different level than images (a)-(c), there was no enhancement on slices adjacent to $(d)$.

Table 1 Changes in the intensity and extent of enhancement of Gd-DTPA enhancing lesions following IVMP

\begin{tabular}{lllll}
\hline & 1 day & 3 days & 1 week & 1 month \\
\hline No enhancement & 15 & 43 & 12 & 56 \\
Intensity: & & 38 & 38 & 22 \\
$\quad$ decreased & 55 & 2 & 26 & 6 \\
unchanged & 14 & 2 & 10 & 1 \\
increased & 1 & 30 & 42 & 22 \\
Extent: & 42 & 10 & 26 & 4 \\
$\quad$ decreased & 27 & 2 & 5 & 3 \\
$\quad$ unchanged & 1 & & \\
\hline
\end{tabular}

(Number of enhancing lesions pre-treatment $=85$ )

Table 2 New MRI activity following IVMP

\begin{tabular}{|c|c|c|c|c|c|}
\hline & 1 day & 3 days & 1 week & 1 month & Total \\
\hline New lesions & 0 & $\mathbf{0}$ & 2 & 30 & 32 \\
\hline Enlarged lesions ${ }^{\star}$ & 0 & 0 & 1 & 20 & 21 \\
\hline $\begin{array}{l}\text { Enhancing lesions }{ }^{\star} \\
\text { (size unchanged) }\end{array}$ & 0 & 0 & 0 & 3 & 3 \\
\hline $\begin{array}{l}\text { †intensity/extent of } \\
\text { enhancement in lesions } \\
\text { which enhanced before IVMP }\end{array}$ & 2 & 1 & 8 & 0 & 11 \\
\hline TOTAL & 2 & 1 & 11 & 53 & 67 \\
\hline
\end{tabular}

^ not enhancing before IVMP activity. Four types of "active" lesions were identified (table 2): 1) New lesions; 2) Lesions which had not enhanced on the first scan, but which subsequently enlarged; 3) Lesions which had not enhanced on the first scan, but which subsequently enhanced without changing size; 4) Lesions which had enhanced on the first scan, and which subsequently showed an increased intensity and/or extent of enhancement.

On entry into the study, a neurological history and examination was performed, and at each follow up, any changes in pre-existing symptoms or new symptoms were noted. The patient was re-examined after one week, or after new symptoms had recurred.

The project was approved by the Joint Medical Ethics Committee of the National Hospital for Neurology and Neurosurgery, and all patients gave informed and written consent.

\section{Results}

MRI

1) Lesions enhancing before treatment (table 1)

At first scan, there was a total of 85 enhancing lesions ( 82 in the brain, and three in the spinal cord). Immediately after the three days of IVKP, 81 lesions (96\%) showed a decrease in BBB abnormality-43 (51\%) no longer enhanced, and $38(45 \%)$ displayed a reduced intensity of enhancement (fig 1). In only three lesions was there an increased intensity and/or extent of enhancement during the three days of treatment (table 2).

After one week, re-enhancement was seen in 33/43 lesions which had ceased enhancing after three days, while 27 other lesions showed more intense enhancement than they did after three days (fig 1). However, when compared with the pre-treatment study, enhancement was less intense (or absent) in 50/85 (59\%) lesions, and less extensive (or absent) in 54/85 (64\%) lesions, while only $10 / 85(12 \%)$ displayed an increased intensity and/or extent of enhancement.

After one month, 29 (34\%) lesions still enhanced, although usually with a reduced intensity and extent compared with the pretreatment study.

Figure 2 illustrates the changing frequency of persisting enhancement in these 85 lesions over the month following IVMP (dashed line). Also shown are the frequencies of persisting enhancement after one month in three recently published serial monthly MRI studies in patients with relapsing-remitting or secondary progressive MS who were not being treated with steroids. ${ }^{15-17}$ In these studies, $26-33 \%$ of lesions displayed persisting enhancement after one month, and the continuous lines define the proportion of lesions expected to display persisting enhancement at any point during the month, assuming a steady rate of decrease in the number of enhancing lesions over this period. For the IVMP treated patients, the proportions of lesions which still enhance is much lower than that predicted in the untrea- 


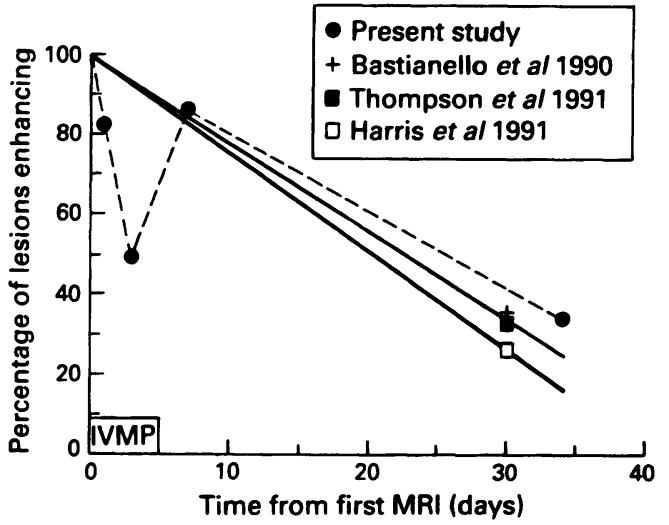

Figure 2 Frequency of persisting Gd-DTPA enhancement in lesions which enhanced prior to treatment with IVMP.

ted patients after one and three days (that is, during the actual period of IVMP treatment), but is similar to that predicted after one week and one month.

\section{2) New disease activity (table 2)}

Sixty seven "active" lesions were identified during the follow up period, of which 53 were first identified at the one month follow up, 11 at one week, and three during treatment with IVMP. The majority of new lesions displayed Gd-DTPA enhancement $(27 / 32 ; 84 \%)$.

CLINICAL

Eight patients improved over the first week. In four patients, recovery was rapid, beginning during the period of treatment, and all had improved substantially after one week; three patients who had been wheelchair bound before treatment were able to walk with assistance, due to recovery of pyramidal function, while one patient who was initially disabled with ataxia, oscillopia, nausea and diplopia due to a pontine lesion had almost completely recovered after one week. In four other patients, the symptomatic and objective improvements were slight and occurred gradually over the course of the week. Two patients, who presented with a purely sensory relapse, were no different after one week, but gradually recovered normal sensation during the remainder of the month.

There were three clinical relapses during the month. Two patients developed sensory symptoms attributable to a new spinal cord lesion three and four weeks following the start of treatment respectively. After an initial gradual improvement, one patient had recurrence of her original spinal cord symptoms with deterioration in mobility three weeks after the start of treatment. No patient deteriorated during the first week after treatment.

CLINICAL-MRI CORRELATIONS

The lesion responsible for the recent clinical relapse was monitored by $M R I$ in four patients- 3 lesions were in the spinal cord, and one in the brain stem (spinal MRI was not performed on the other six patients in whom the recent relapse was attributable to a cord lesion). All four symptomatic lesions displayed enhancement on the pre-treatment scan (the remaining 81 enhancing lesions seen at this time were asymptomatic). The four lesions each showed marked or total reduction of enhancement after three days of treatment, followed by re-enhancement after one week, which was less intense than before treatment in two lesions, of similar intensity in one and more intense in one. Over the first week, two patients experienced a substantial and sustained clinical improvement (enhancement in the symptomatic lesion after one week was less intense than pretreatment in one and more intense in the other), one had improved slightly (enhancement in the symptomatic lesion after one week was the same intensity as pretreatment), and one remained clinically unchanged (enhancement was less intense after one week).

After one month, one of four lesions still enhanced, and this patient experienced a return of the original symptoms one week before the follow up scan; the other three patients were all clinically much improved.

\section{Discussion}

Several lines of evidence suggest that the presence of BBB abnormality in MS lesions (detected by Gd-DTPA enhancement) is of functional significance. First, Gd-DTPA enhancement correlates with perivascular inflammation, ${ }^{18}$ a hallmark of actively demyelinating lesions. Secondly, in relapsing-remitting and secondary progressive MS, new asymptomatic cerebral MRI lesions usually display GdDTPA enhancement for two to six weeks, which is similar to the time course of clinical relapses. ${ }^{1215-1719}$

Thirdly, a recent serial study of the symptomatic lesion in acute optic neuritis demonstrated a close temporal association between the presence of enhancement and the acute functional deficit: thus Gd-DTPA-enhancement was a consistent feature of the early lesion, ${ }^{20}$ at which time there was defective visual acuity and low amplitude visual evoked potentials (VEP) indicating conduction block; one month later, without treatment, enhancement had ceased, visual acuity had returned to normal, and there had been a significant increase in the amplitude of the VEP, indicating improved conduction, in spite of persisting demyelination (prolonged latency of the VEP). ${ }^{21}$

How might BBB abnormalities produce clinical deficit? Several factors may be relevant. First, oedema per se may have a direct effect on conduction either through alteration in the axonal environment or by compression compromising the local blood supply in parts of the nervous system where axons are tightly packed and expansion is restricted (for example, optic nerves, brain stem, spinal cord). Secondly, normal serum constituents, in particular complement, can produce sub-lethal and lethal damage to oligodendrocytes, ${ }^{22}$ which could lead to demyelination. Thirdly, it is possible 
that many of the immunologically active cells which are seen in acute/enhancing MS lesions, and which probably mediate the demyelination, have originated in the periphery, ${ }^{23}$ and enter the CNS through the damaged BBB. Fourthly, cytokines released by the inflammatory cells might have a direct effect on the excitable membrane of the axons as they do on muscle. ${ }^{24}$

This study shows that IVMP consistently and rapidly reduces BBB abnormalities in the great majority of enhancing MS lesions, and it is therefore possible that this mechanism contributes to the accelerated recovery from acute relapses associated with IVMP treatment.

However, our study also shows an apparent discordance between BBB changes and the clinical course in that, after one week, many lesions displayed increased enhancement in comparison with day three, while at the same time all patients were clinically stable or improving. Specifically, this evolution was seen in the four symptomatic lesions studied by MRI. Two possible explanations for this discordance deserve mention. First, it is quite possible that there are pathophysiological mechanisms in MS relapses which are independent of the $B B B$, and which may or may not be modified by steroids, for example, immunological changes. Secondly, the BBB abnormalities after one week often appeared to be less marked than before IVMP treatment and it is possible that the degree of recurrent BBB impairment and its attendant effects was not sufficient to cause further clinical deterioration. To clarify the inter-relationship of steroid therapy, BBB status and clinical deficit more precisely, future studies should concentrate on acute symptomatic lesions using NMR techniques which quantitate BBB permeability. ${ }^{25}$

The re-enhancement seen within a few days of stopping treatment also raises the question of whether a longer course of steroids should be used in acute relapses with the aim of suppressing enhancement in a symptomatic lesion until it would naturally have ceased (usually after two to six weeks). However, such an approach would seem no more likely to prevent the development of new disease activity once treatment was stopped-in this study, we frequently found new lesions in the month followihg a three day course of IVMP, and new lesions have also been seen within a few days of completing a 10 day course of high dose IVMP. ${ }^{26}$ We believe that the decision to treat should be based on clinical criteria alone, and should not be influenced by asymptomatic MRI-detected disease activity, the prognostic significance of which is uncertain. In this study, all patients had symptomatic improvement in the initial weeks following treatment, and in our experience, it is unusual for the original symptoms to recur within such a period following a three day course of IVMP. It therefore remains our practice to limit treatment to the three day course, given that side effects become increasingly likely with increasing duration of treatment. $^{2728}$
The MRI scanner used in this study was provided by the Multiple Sclerosis Society of Great Britain and Northern Ireland.

1 Miller H, Newell DJ, Ridley A. Multiple sclerosis: treatment of acute exacerbations with corticotropin (ACTH). Lancet $1961 ; \mathbf{i i}: 1120-2$.

2 Rose AS, Kuzma JW, Kurtke JF, et al. Co-operative study in the evaluation of therapy in multiple sclerosis: ACTH versus placebo. Neurology 1970;5:1-59.

3 Barnes MP, Bateman DE, Cleland PG, et al. Intravenous methylprednisolone for multiple sclerosis in relapse. I Neurol Neurosurg Psychiatry 1985;48:157-9.

4 Durelli L, Cocito D, Riccio A, et al. High-dose intravenous methylprednisolone in the treatment of multiple sclerosis: clinical-immunologica correlations. Neurology 1986;36: 238-43.

5 Milligan NM, Newcombe R, Compston DAS. A doubleblind controlled trial of high dose methylprednisolone in patients with multiple sclerosis. 1. Clinical effects. $f$ patients with multiple sclerosis. 1. Clinical

6 Thompson AJ, Kennard C, Swash M, et al. Intravenous methylprednisolone and ACTH in the treatment of acute relapse in multiple sclerosis. Neurology 1989;39:969-71.

7 Kesselring J, Miller DH, MacManus DG, et al. Quantitative magnetic resonance imaging in multiple sclerosis: the effect of high dose intravenous methylprednisolone. $\mathcal{F}$ Neurol Neurosurg Psychiatry 1989;52:14-7.

8 Compston DAS, Milligan NM, Hughes PJ, et al. A doubleblind controlled trial of high dose methylprednisolone in patients with multiple sclerosis. 2. Laboratory results. Neurol Neurosurg Psychiatry 1987;50:517-22.

9 Triarhou LC, Herndon RM. The effect of dexamethasone on L-alpha-lysophosphatidylcholine (lysolecithin)induced demyelination of the rat spinal cord. Arch Neurol 1986;43:121-5.

10 Kermode AG, Thompson AJ, Tofts PS, et al. Breakdown of the blood-brain barrier precedes symptoms and other MRI signs of new lesions in multiple sclerosis. Brain 1990;113:1477-89.

11 Troiano R, Hafstein $M$, Ruderman M, Dowling P, Cook S. Effect of high-dose intravenous steroid administration on contrast-enhancing computed tomography scan lesions in multiple sclerosis. Ann Neurol 1984;15:257-63.

12 Miller DH, Rudge P, Johnson G, et al. Serial gadolinium enhanced magnetic resonance imaging in multiple sclerosis. Brain 1988;111:927-39.

13 Kermode AG, Tofts PS, Thompson AJ, et al. Heterogeneity of blood-brain barrier changes in multiple sclerosis: an MRI study with gadolinium-DTPA enhancement. Neurology 1990;40:229-35.

14 Poser CM, Paty DW, Scheinberg LC, et al. New diagnostic criteria for multiple sclerosis: guidelines for research protocols. Ann Neurol 1983;13:227-31.

15 Bastianello S, Pozzilli C, Bernadi S, et al. Serial study of gadolinium-DTPA MRI enhancement in multiple sclerosis. Neurology 1990;40:591-5.

16 Thompson AJ, Kermode AG, Wicks D, et al. Major differences in the dynamics of primary and secondary progressive multiple sclerosis. Ann Neurol 1991;29: 53-62.

17 Harris JO, Frank JA, Patronas NJ, McFarlin DE, McFarland HF. Serial gadolinium enhanced magnetic resonance imaging scans in patients with early relapsing-remitting multiple sclerosis: implications for clinical trials and natural history. Ann Neurol 1991;29:548-55.

18 Katz J, Taubenberger J, Raine C, et al. Gadolinium enhancing lesion on magnetic resonance imaging: neuropathological findings. Ann Neurol 1990;28:243.

19 Kappos L, Gold R, Hofmann E, Keil W, Clauss W. Multiple sclerosis: diagnostic criteria and the role of contrast sclerosis: diagnostic criteria and the role of contrast enhs. Contrast Media in MRI. Bussum: Medicom Europe, eds. Contrast $M$.

20 Youl BD, Miller DH, MacManus DG, et al. Optic nerves show gadolinium enhancement in early optic neuritis. Neuroradiology 1991;33(suppl): 129-30.

21 Youl BD, Turano G, Miller DH, et al. The pathology of acute optic neuritis: an association of gadolinium leakage with clinical and electrophysiological deficits. Brain 1991; 114:2437-50.

22 Compston DAS. The dissemination of multiple sclerosis. $\mathcal{F}$ Roy Coll Phys London 1990;24:207-18.

23 Hafler DA, Weiner HL. In vivo labelling of blood T cells; rapid trafficking to cerebrospinal fluid in multiple sclerosis. Ann Neurol 1987;22:89-93.

24 Tracey KJ, Lowry SF, Beutler B, Cerami A, Albert JD, Shires GT. Cachectin/tumour necrosis factor mediates Shires GT. Cachectin/tumour necrosis factor mediates
changes of skeletal muscle plasma membrane potential. $\mathcal{f}$ changes of skeletal muscle p

25 Tofts PS, Kermode AG. Measurement of blood-brain barrier permeability and leakage space using dynamic MR imaging. 1. Fundamental Concepts. Magn Reson Med 1991;17:357-67.

26 Barkhof F, Hommes O, Scheltens Ph, Valk J. Quantitative MR changes in gadolinium-DTPA enhancement after high dose intravenous methylprednisolone in multiple sclerosis. Neurology 1991;41:1219-22.

27 Felson DT, Anderson JJ. A cross-over evaluation of association between steroid dose and bolus steroids and avascular necrosis of bone. Lancet 1987; i: $902-5$.

28 Warren KG, Catz I, Jeffrey VM, Caroll DJ. Effect of methylprednisolone on CSF IgG parameters, myelin basic protein, and anti-myelin basic protein in multiple sclerosis exacerbations. Can $\mathcal{f}$ Neurol Sci 1986;13:23-30. 